\title{
Analyse von Ausaperungsmustern der saisonalen Schneedecke mit Fernerkundungsmethoden
}

\begin{abstract}
Remote sensing techniques allow to monitor the snow cover distribution during the melting season and is used to study the ablation process. Three Alpine basins in Switzerland have been selected in order to evaluate regional differences in the North-South and West-East direction. The variing snow cover distribution pattern during the melting process was derived from Landsat-MSS, -TM and SPOT-XS images. Utilizing a Geographical Information System (GIS) additional features influencing the snow distribution, such as glaciers, permafrost, forest and vegetation, have been taken into account and form a set of Snow Cover Units (SCU). In addition SCU maps were developed enabling an enhanced analysis of the snow cover distribution with regard to the mentioned ground properties. The mutual effect of snow cover and permafrost has been studied.
\end{abstract}

\section{Einleitung}

Die alpine Schneedecke ist durch ein ungleichmäßiges Verteilungsmuster sowie eine große Variabilität bezüglich Schichtung, Höhe und Wasseräquivalent (SCHWEIZER 1996) gekennzeichnet. Anhand von Punktmessungen oder Transekts wurde die Verteilung schon in vielen Arbeiten untersucht. KILLINGTVEID und SAND (1991) ermittelten mit Hilfe von Schneekurven eine Schneeverteilungsfunktion und verbesserten damit die Abflußmodellierung. WITMER (1982) entwickelte eine Methode, mit der auf der Basis von Schneemessungen die Schneehöhen flächendeckend kartiert werden können. Aber auch mit ausgedehnten Feldmeßkampagnen werden nicht alle Geländeformen und Höhenbereiche umfassend abgedeckt, und es wird nur eine verhältnismäßig kleine Anzahl von Stichproben gewonnen. Es bleibt also immer eine Unsicherheit, ob die Variabilität vollständig erfaßt werden konnte.

Die satellitengestützte Fernerkundung bietet eine gute Möglichkeit, die Schneedecke flächenhaft zu beobachten. Mit hochauflösenden, optischen Sensoren der Erderkundungssatelliten kann sie heute operationell kartiert werden (DOZIER 1989, ROSENTHAL 1996). Die Schneehöhe oder das Wasseräquivalent kann aber nur mit Hilfe von Modellen aus den Schneeabnahmekurven (siehe Abb. 1) abgeleitet werden (HALL und MARTINEC 1985, SEIDEL et al. 1996). Das Ausaperungsmuster, welches mit Zeitreihen von Satellitenaufnahmen erfaßt werden kann, ermöglicht ferner Rückschlüsse auf die Verteilung der saisonalen Schneedecke. Durch die Übernahme der
Schneekartierungen in ein Geographisches Informationssystem (GIS) kann das Ausaperungsmuster nach verschiedenen schneerelevanten Kriterien analysiert werden (EHRLER und SEIDEL 1995).

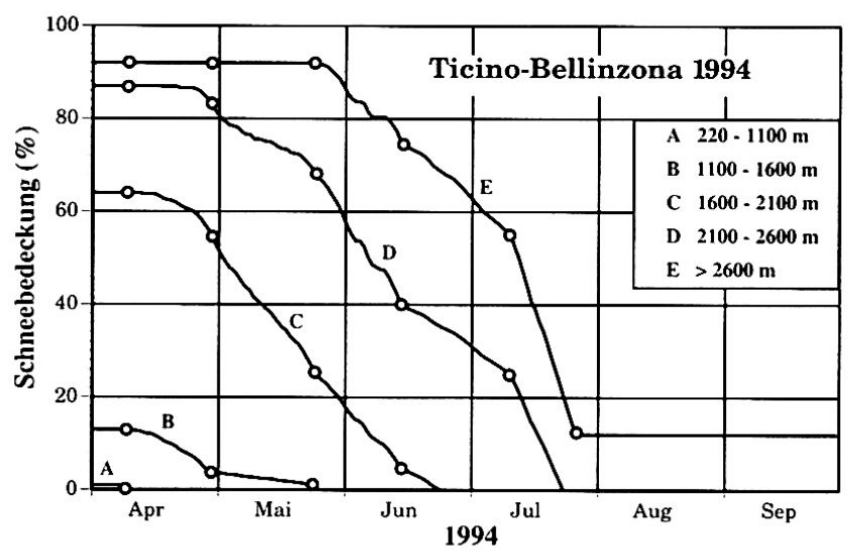

Abb.1 Schneeabnahmekurven mit Aufnahmezeitpunkten am Beispiel des Einzugsgebietes Ticino-Bellinzona 1994. Höhenzonen A-E.

\section{Die Untersuchungsgebiete}

Drei hochalpine Einzugsgebiete (siehe Abb. 2), die in verschiedenen Klimaregionen liegen, wurden ausgewählt, um die Variabilität innerhalb der schweizerischen Alpen zu erfassen:

- Das Einzugsgebiet des Rheins bis Felsberg $(3250$ km², 560-3614 m ü. M., Vergletscherung $64 \mathrm{~km}^{2}$ ) wurde in fünf Höhenzonen unterteilt. Von diesem Untersuchungsgebiet werden seit 1982 satellitengestützte Schneekartierungen gewonnen, so daß eine mehrjährige Datenreihe ausgewertet werden konnte.

Cornel Ehrler, Jesko Schaper, Institut für Kommunikationstechnik, Bildwissenschaft, ETH Zürich (cornel,jschaper)@vision.ee.ethz.ch 
- Das Einzugsgebiet der Rhone bis Sion $\left(3371 \mathrm{~km}^{2}\right.$, 491-4634 m ü. M., Vergletscherung $580 \mathrm{~km}^{2}$ ) ist charakterisiert durch eine hohe Vergletscherung von $17 \%$. Es wurde wegen der großen Höhendifferenz in sieben Höhenzonen unterteilt.

- Das südwärts orientierte Einzugsgebiet des Ticino bis Bellinzona hat eine Fläche von $1532 \mathrm{~km}^{2}$, erstreckt sich über einen Bereich von 220-3402 $\mathrm{m}$ ü. M. und ist nur minimal vergletschert $\left(8 \mathrm{~km}^{2}\right)$. Es wurde, wie RheinFelsberg, in fünf Höhenzonen unterteilt.

Diese drei Einzugsgebiete werden weiter untergliedert in Regionen mit homogener Schneelage, die behelfsmäßig benannt werden ("Gotthard und Tödi», "Goms» usw.).

\section{Variabilität der Schneedecke}

Betrachtet man die Schneebedeckung in den Alpen, so dominiert die Zunahme mit der Höhe. Im Laufe eines Winters bilden sich als Folge eines komplexen Prozesses jedoch auf gleicher Höhenlage enorme Unterschiede hinsichtlich Schneehöhe und -bedeckungsgrad aus. Die räumliche und zeitliche Verteilung von Schnee in einem Gebiet läßt sich als eine Funktion des Schneefalls, der Umverteilung und der Ablation charakterisieren. Die Differenzierung findet innerhalb von wenigen Zentimetern bis zu Hunderten von Kilometern statt. Die großräumige $(>10 \mathrm{~km})$ Variabilität wird durch die typischen
Wetterlagen einer Region bestimmt. Die Schneefälle, der "Input» der Schneedecke, fallen von Region zu Region sehr unterschiedlich aus.

Alle anderen Heterogenitäten sind mittel- $(100 \mathrm{~m}$ bis $10 \mathrm{~km}$ ) oder kleinräumig $(<100 \mathrm{~m})$ und haben mehrere Ursachen. Der Schnee haftet schlecht auf Steilhängen (Hangneigung $>45-50^{\circ}$ ) und stürzt meist bereits während des Schneefalls als Lockerschneelawine den Hang hinunter. Bei geringerer Hangneigung bildet sich eine kompakte Schneedecke, die aber auch der Schwerkraft ausgesetzt ist und als Lawine, Schneerutsch oder schleichend (Schneekriechen) hangabwärts in Bewegung gerät.

Der Wind lagert vor allem oberhalb der Waldgrenze den Schnee in großem Maße um. Über Gebirgskämme und Pässe findet eine Umverteilung vom Luv ins Lee statt, und kleinräumig wird der Schnee von Kuppen und Rükken abgeblasen und in Mulden deponiert.

Die Beschaffenheit der Schneeunterlage und speziell der Wald beeinflussen ebenfalls die Bildung und die Ablation der Schneedecke. Im geschlossenen Wald fällt weniger Schnee auf den Boden, doch ist er dort geschützt vor Wind und Sonneneinstrahlung, und die Ausaperung trotz einer allgemein geringeren Schneehöhe kann sich deshalb verzögern. Im lockeren Wald und in Lichtungen kann die Schneedecke sogar mächtiger sein als im offenen Gebiet.

Der Wärmefluß vom Boden in die Schneedecke ist von der Unterlage abhängig. Der Schnee sollte sich deshalb

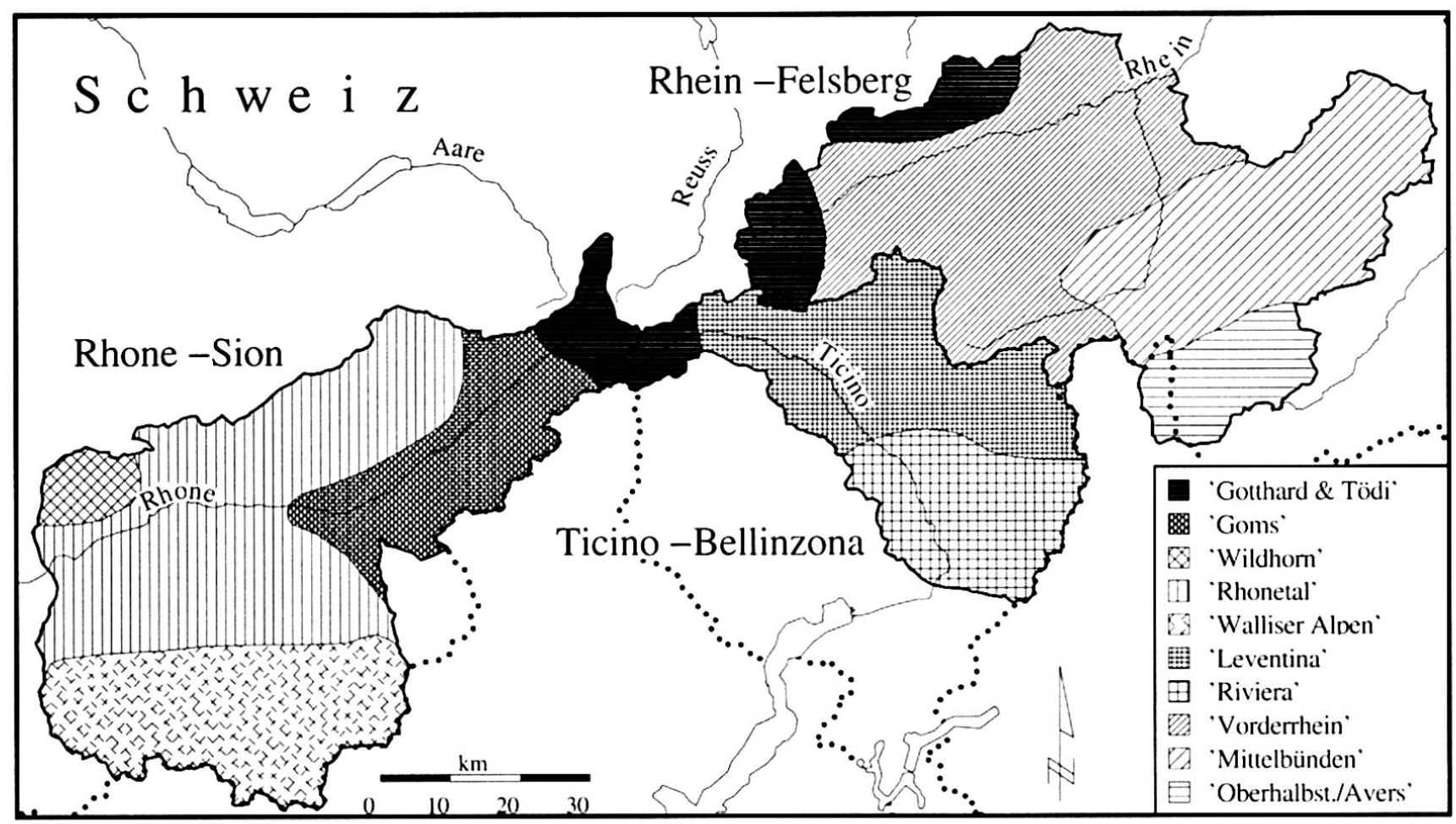

Abb. 2 Die Untersuchungsgebiete Rhein-Felsberg. Rhone-Sion und Ticino-Bellinzona, unterteilt in Regionen mit homogener Schneelage. 
auf Gletscher oder Permafrostboden länger halten als auf eisfreien Böden.

Letztlich bringt die Schneeschmelze eine weitere Variabilität mit sich. Die direkte Sonneneinstrahlung beschleunigt die Ablation. Sie ist abhängig vom lokalen Einfallswinkel und vom Horizontverlauf.

\section{Schneekartierung mit Satellitendaten}

Satelliten eignen sich in besonderer Weise zu langandauernden, periodischen Überwachungen und Kartierungen von großen, abgelegenen oder schwer zugänglichen Gebieten. Vorteilhaft ist, daß das Meßobjekt von oben in seiner flächenhaften Ausdehnung erfaßt wird. Für die Erkennung und Differenzierung von Schnee und seiner Umgebung sind in erster Linie Sensortypen geeignet, die im sichtbaren Wellenlängenbereich und im nahen Infrarot arbeiten (RANGO 1985). Außerdem ist für die Erfassung der kleinräumigen Ausaperungsprozesse eine hohe geometrische Auflösung von 10-100 m, wie sie bei Landsat- und SPOT-Satelliten gegeben ist, von großem Nutzen. In der vorliegenden Arbeit wurden deshalb LandsatMSS, Landsat-TM und SPOT-XS-Daten verwendet.

Alle ausgewählten Aufnahmen wurden georeferenziert und, wenn nötig, im Falle einer großen Reliefverzerrung mit einem digitalen Höhenmodell orthorektifiziert. Die multispektralen Datensätze wurden bezüglich Schneebedeckung (Schnee, Übergangszone, aper) thematisch ausgewertet unter Berücksichtigung der topographiebedingten Beleuchtungsunterschiede, um eine korrekte Interpretation sowohl in Schattenlagen als auch an sonnenabgewandten Hängen zu garantieren (EHRLER und SEIDEL 1995). Im stark vergletscherten Einzugsgebiet der Rhone ist zusätzlich auch blankes Gletschereis kartiert worden. Das detaillierte Klassifikationsschema kann der Abb. 3 entnommen werden.

Für die Untersuchung wurden nur Aufnahmen von Satellitenüberflügen verwendet, denen keine Schneefälle unmittelbar vorangegangen waren; der Neuschnee verwischt das differenzierte Ausaperungsmuster der saisonalen Schneedecke.

\section{Das Konzept der Schneebedeckungseinheiten (SCU)}

Um das Ausaperungsmuster der Schneekartierungen erfassen und analysieren zu können, müssen die physikalischen Ursachen der Variabilität in räumliche Einheiten umgesetzt werden. In Anlehnung an den in hydrologischen Modellen verwendeten Ansatz der Hydrological Response Unit (HRU), der ein Gebiet in Einheiten gleichen hydrologischen Verhaltens unterteilt, wurde die Schneebedeckungseinheit oder Snow Cover Unit (SCU) eingeführt. Eine Schneebedeckungseinheit ist eine Verei-

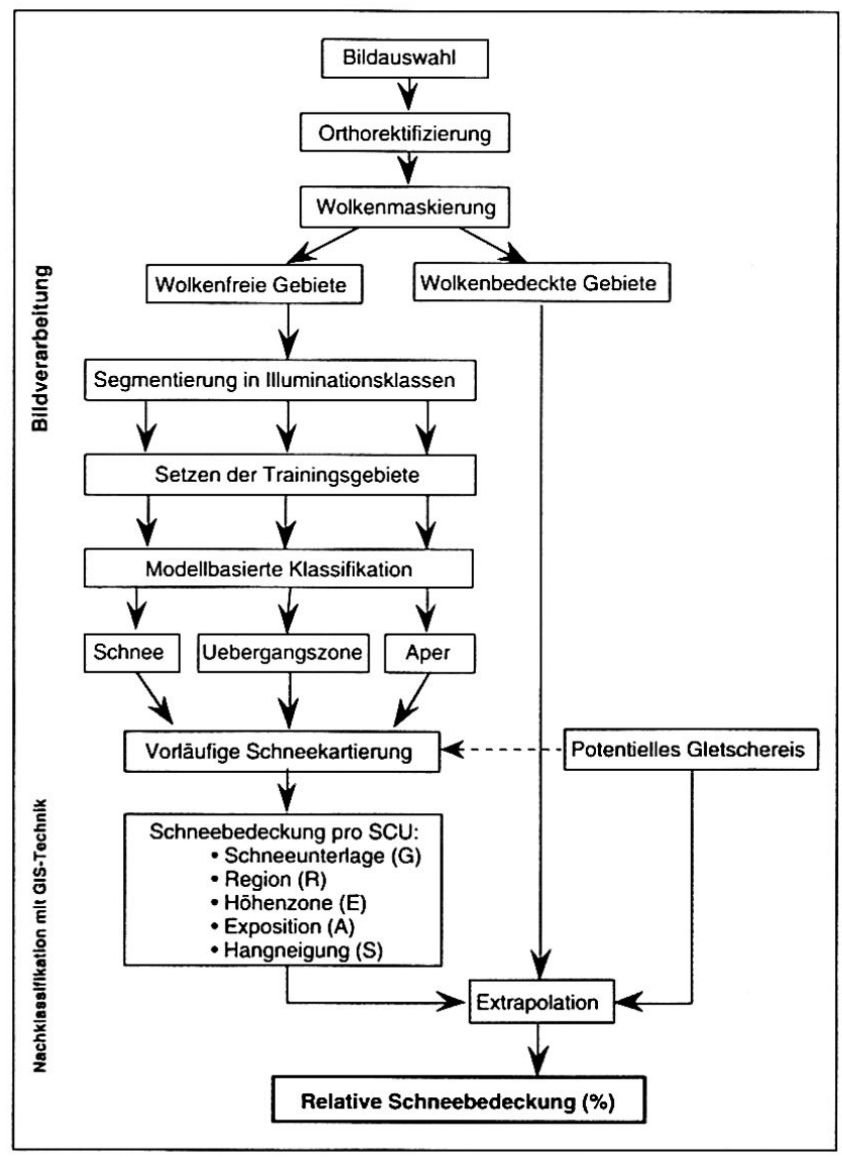

Abb.3 Schneeklassifikationsschema für optische Sensoren.

nigung von räumlich verstreuten Flächen mit gleicher Schneebedeckung. Die vorher erwähnten Gegebenheiten, die die Schneedecke beeinflussen, sind als einzelne Ebenen in einem GIS zusammengetragen worden:

- Schneeunterlage (G: ground), $\mathrm{N}_{\mathrm{G}}=5$ Klassen: Gletscher, Permafrost, Vegetationslos, Wald, Restklasse

- Regionen mit gleichen Wetterlagen (R: region), $\mathrm{N}_{\mathrm{R}}=$ 3-5 Klassen pro Untersuchungsgebiet

- Höhenzonen (E: elevation), $\mathrm{N}_{\mathrm{E}}=5-7$ Klassen, im Normalfall mit Höhenbereich von $500 \mathrm{~m}$

- Exposition (A: aspect), $\mathrm{N}_{\mathrm{A}}=8 \mathrm{Klassen}$ : Nord, Nordost, Ost, Südost, Süd, Südwest, West, Nordwest

- Hangneigung (S: slope), $\mathrm{N}_{\mathrm{S}}=5$ Klassen: flach ohne Exposition $\left(<8^{\circ}\right), 9-18^{\circ}, 19-27^{\circ}, 28-35^{\circ}, \geq 36^{\circ}$

Die Ebenen Höhenzone, Exposition und Hangneigung sind aus feinmaschigen Höhenmodellen mit größtenteils 25-m-Auflösung abgeleitet worden. Die Rasterweite der Schneebedeckungseinheit wurde daher auf $25 \mathrm{~m}$ bzw. bei Rhein-Felsberg auf $100 \mathrm{~m}$ festgelegt. In Satellitenbildern ist die Schneedecke im Wald nur teilweise sichtbar. Eine Voruntersuchung ergab, daß sich innerhalb der zehn Waldklassen der schweizerischen Arealstatistik bezüglich Schneebedeckung nur geringe Unterschiede zeigen 
und sie zu einer Klasse Wald zusammengefaßt werden können. Die Klasse Permafrost wurde mit dem Modell Permakart (KELLER 1994) berechnet. Im Höhenbereich zwischen $2000 \mathrm{~m}$ und $3000 \mathrm{~m}$ ist das Fehlen der Vegetation ein guter Hinweis auf das Vorhandensein von Permafrost. Daher wurde mit Hilfe der Arealstatistik zusätzlich die Klasse Vegetationslos gebildet, um zu sehen, ob sie sich bezüglich Schneebedeckung wirklich ähnlich verhält wie die Klasse Permafrost. Die Schneebedekkungseinheiten sind nun durch die Überlagerung aller Ebenen gebildet worden. Die feine Untergliederung führt zu einer großen Anzahl von Schneebedeckungseinheiten. Die potentielle Anzahl pro Untersuchungsgebiet berechnet sich wie folgt:

$$
N_{S C U}=N_{G} \cdot N_{R} \cdot N_{E} \cdot\left[N_{A}\left(N_{S}-1\right)+1\right]
$$

Nicht alle Kombinationen kommen vor, so daß sich die Anzahl der Schneebedeckungseinheiten deutlich reduziert auf 1381 in Ticino-Bellinzona, 1781 in Rhein-Felsberg und 2935 in Rhone-Sion. Die mittlere Fläche der Schneebedeckungseinheit beträgt etwa $1 \mathrm{~km}^{2}$ bzw. im Einzugsgebiet Rhein-Felsberg knapp $2 \mathrm{~km}^{2}$. Die Schneekartierungen wurden in das GIS übertragen und anhand der Schneebedeckungseinheiten ausgewertet.

\section{Die Ausaperung innerhalb einer Höhenzone}

In einem alpinen Einzugsgebiet senken sich die Schneebedeckungskurven in den tieferen Höhenzonen früher als in den oberen, wie es Abbildung 1 am Beispiel des Einzugsgebiets Ticino-Bellinzona zeigt. Dies ist durch die höhenabhängigen Temperatur- und Niederschlagsunterschiede verursacht und entspricht den Erwartungen. Zusätzlich verzögern in hochgelegenen Gebieten die Schneefälle im Sommerhalbjahr die Ausaperung, weil sie die zu schmelzende Schneemenge vergrößern und die Schmelzraten durch eine höhere Albedo reduzieren.

Der Ausaperungsprozeß innerhalb einer Höhenzone ist aber durch die Variabilität der Schneedecke bestimmt und kommt in den Schneekartierungen deutlich zum Vorschein. Die Ergebnisse werden am Beispiel der Höhenzone D (2100-2600 m) vorgestellt. Es wurden nur Aufnahmen, bei denen die Ausaperung im Gange ist (Schneebedeckung zwischen 10 und 90\%), in die Berechnung einbezogen. Im Einzugsgebiet Rhein-Felsberg konnte eine sechsjährige Reihe ausgewertet werden, bei den restlichen zwei Untersuchungsgebieten handelt es sich um einjährige. Die Tabelle 1 gibt eine Übersicht über die verwendeten Datensätze.

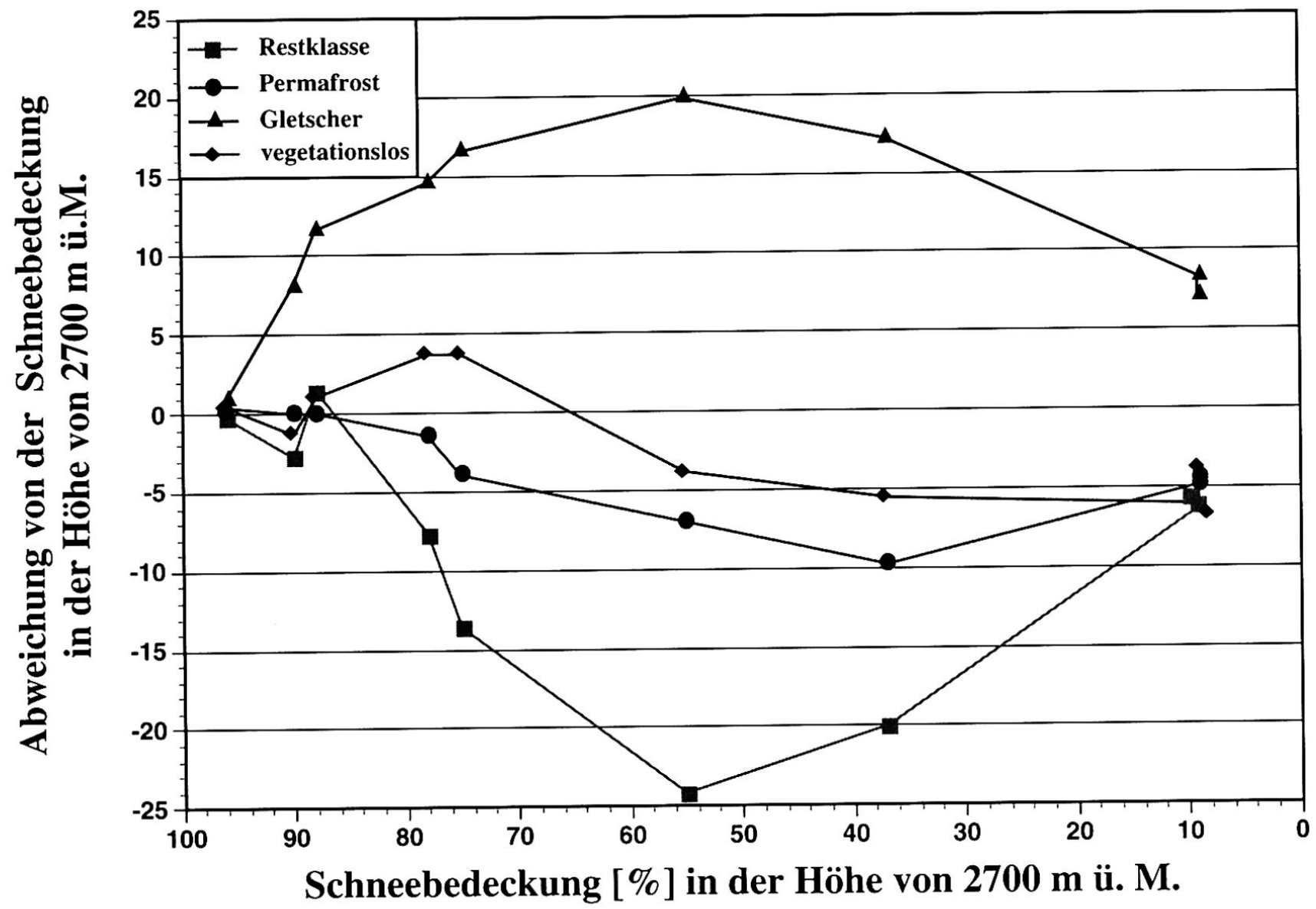

Abb. 4 Effekt der Schneeunterlage: Abweichung der Schneebedeckung auf verschiedenen Unterlagen vom Mittel, interpoliert auf 2700 m ü. M., Einzugsgebiet Rhone-Sion, 1985. 
Die Schneedecke hält sich auf Gletschern deutlich länger als bei den anderen Klassen der Schneeunterlage. Beispielsweise sind bei einer Aufnahme des Einzugsgebiets Rhone-Sion die Gletscher auf der Höhe von $2700 \mathrm{~m}$ ü. M. noch zu $75 \%$ schneebedeckt, während die mittlere Schneebedeckung auf dieser Höhenlage nur 55\% beträgt. Die Schneebedeckung auf den Gletschern weicht also um $+20 \%$ ab. Diese Abweichungen der verschiedenen Schneeunterlageklassen vom Mittel der einzelnen Aufnahmen sind in Abbildung 4 dargestellt: Permafrostböden und vegetationsfreie Gebiete verhalten sich sehr ähnlich und apern früher aus als die Gletscher, aber später als die übrigen, in der Restklasse zusammengefaßten Flächen. Vegetationsfreie Flächen sind in dieser Höhenlage ein Hinweis auf Permafrost.

Die folgenden Abbildungen zeigen alle Ergebnisse vom Einzugsgebiet Rhein-Felsberg. Die sechsjährige Reihe Rhein-Felsberg enthält sowohl Jahre mit einer geringen Schneedecke (Winter 1988/89) wie auch schneereiche Jahre (Winter 1981/82). Damit läßt sich überprüfen, ob das Ausaperungsmuster von Jahr zu Jahr oder je nach Schneereichtum variiert. Alle Daten dieser Reihe sind in den Abbildungen zusammengefaßt, indem die Aufnahmen auf der $x$-Achse sortiert nach ihrer relativen Schneebedeckung in der Höhenzone aufgetragen und die Abweichungen der einzelnen Aggregationen von Schneebedeckungseinheiten zu dieser mittleren Schneebedekkung auf der $y$-Achse abzulesen sind.

Die regionalen Unterschiede der Schneebedeckung innerhalb eines Einzugsgebietes sind beträchtlich: So apert das Gotthardgebiet deutlich später aus als die Gebiete weiter östlich davon, wie in Abbildung 5 zu ersehen ist. Auch die anderen zwei Untersuchungsgebiete grenzen ans Gotthardgebiet und zeigen dasselbe Ergebnis. Dies kann nur mit häufigeren und ausgiebigeren Schneefällen im Gotthardgebiet erklärt werden. Die großen regionalen Unterschiede (vgl. Tabelle 2) bestätigen die Notwendigkeit der Unterteilung in Subregionen (siehe Abb. 2).

Der Effekt der Exposition ist in Abbildung 6 illustriert. Die relative Schneebedeckung ist im Einzugsgebiet Rhein-Felsberg an den ostwärts geneigten Hängen deutlich höher als in den entsprechenden Westhängen, obwohl die direkte Sonneneinstrahlung gleich ist. Die Hauptwindrichtung muß diese beträchtlichen Unter-

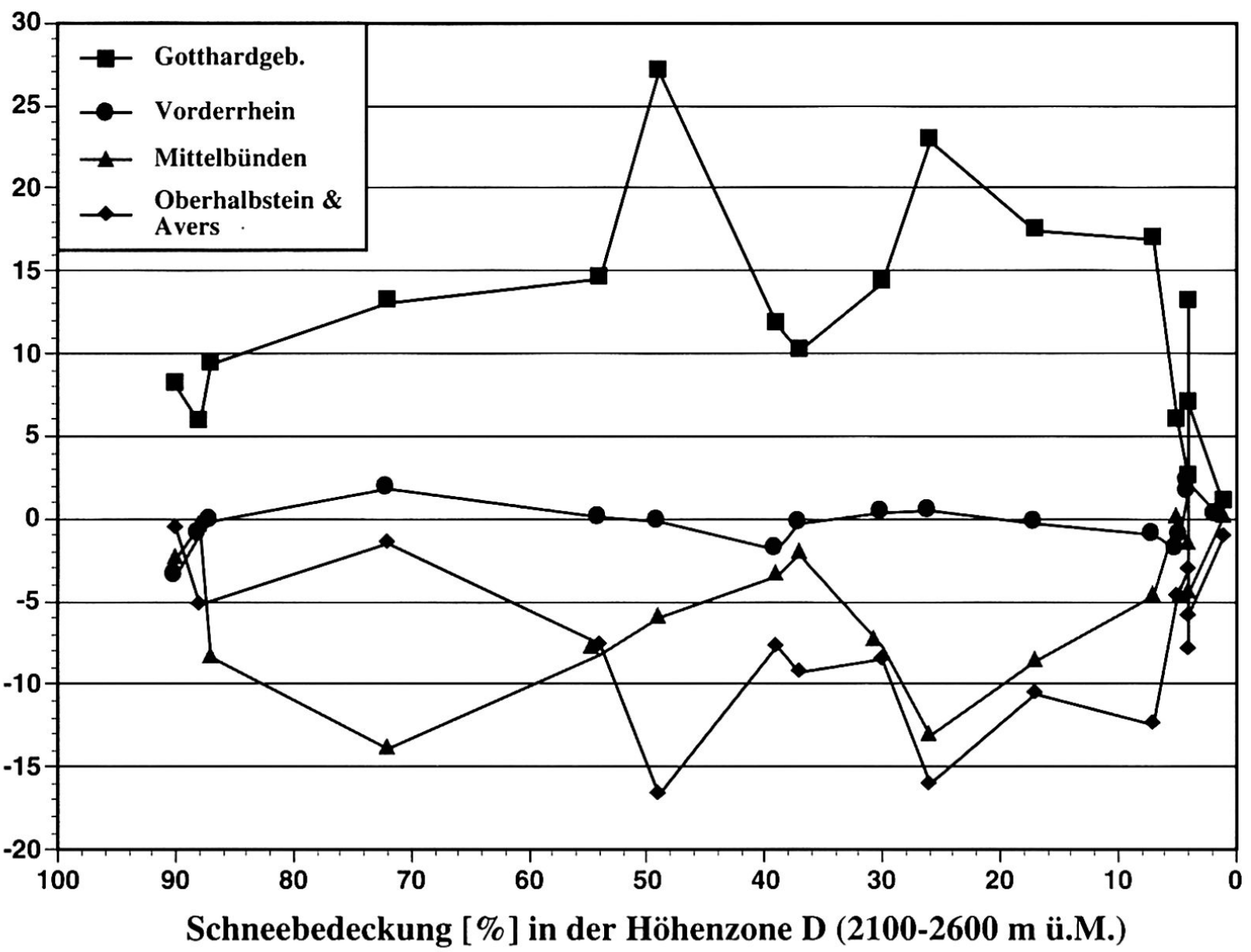

Abb. 5 Effekt der regionalen Variabilität: Abweichung der regionalen Schneebedeckung vom Mittel in der Höhenzone D, Einzugsgebiet Rhein-Felsberg, sechsjährige Reihe. 


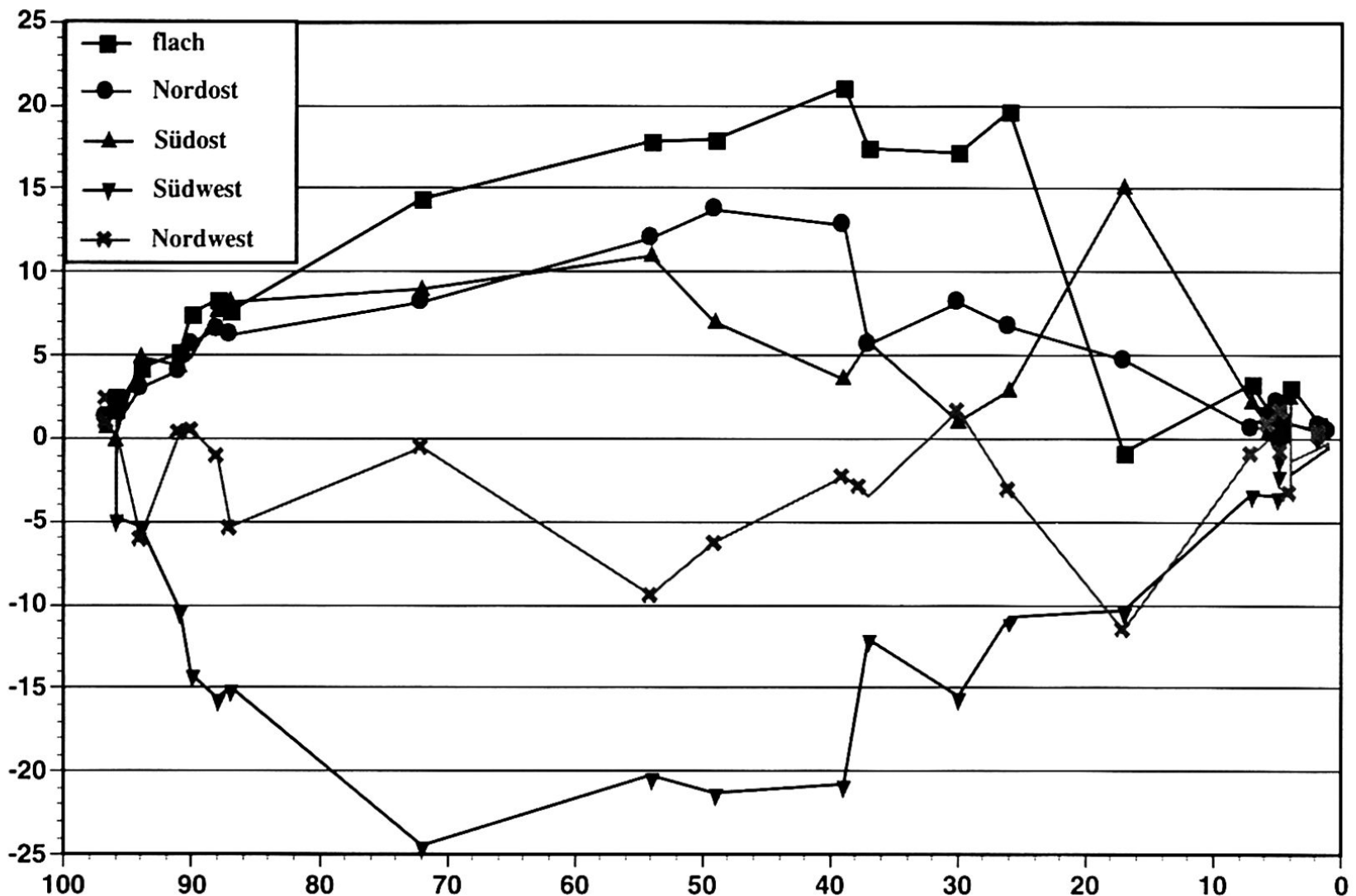

Schneebedeckung [\%] in der Höhenzone D (2100-2600 m ü. M.)

Abb. 6 Effekt der Exposition:Abweichung der Schneebedeckung nach Expositionsklassen vom Mittel in der Höhenzone D, Einzugsgebiet Rhein-Felsberg, sechsjährige Reihe.

schiede größtenteils verursachen, auch wenn andere Einflüsse wie Fehlklassifikationen im sonnenabgewandten Westhang nicht völlig auszuschließen sind. Bei kräftigem Wind liegen die Osthänge also mehrheitlich im Lee, so daß sich dort viel Treibschnee ablagert. Da die Hauptanströmung in den drei Einzugsgebieten nicht gleich ist, sollte sich das in den Treibschneeablagerungen bzw. im Ausaperungsmuster auswirken. Tatsächlich läßt die Schneebedeckung nach Expositionsklassen darauf schließen, daß in Rhein-Felsberg West-, in Rhone-Sion Westsüdwest- und im Tessin Südsüdwestwinde vorherrschen (vgl. Tabelle 2).

Die Hangneigung spielt eine wichtige Rolle, wie Abbildung 7 zeigt. Auf horizontalen Flächen (Hangneigung $<8^{\circ}$ ) ist die relative Schneebedeckung mit Ausnahme des Tessins deutlich höher. Vor allem Triebschnee, aber auch alle Arten von Lawinen lagern sich am Hangfuß ab. Der Trend träte wahrscheinlich noch deutlicher hervor, wenn die Hangneigungsklassen zusätzlich in konkave und konvexe Formen unterteilt würden, weil deren kleinräumiger Umlagerungseffekt den generellen Hangneigungseffekt etwas verwischt.
Die Kurven in den Abbildungen 4, 6 und 7 verlaufen ruhig und kreuzen sich höchst selten, obwohl sie Daten von verschiedenen Jahren enthalten. Sie weisen darauf hin, daß sich das Ausaperungsmuster jedes Jahr wiederholt, unabhängig vom Schneereichtum.

Die gesamthafte Betrachtung der Resultate aller drei Einzugsgebiete ermöglicht es, die verschiedenen Faktoren, die die Variabilität der Schneedecke verursachen, gewichten zu können. Für jedes Einzugsgebiet wurde das arithmetische Mittel der prozentualen Schneebedekkung aller Schneebedeckungseinheiten von allen verwendeten Aufnahmen berechnet (siehe Tab. 1). Die Abweichungen der einzelnen Aggregationen von Schneebedeckungsklassen zu diesem Mittel sind in Tabelle 2 dargestellt. Da die räumliche Verteilung und die Fläche einer Schneebedeckungseinheit nicht zufällig sind - Gletscher kommen vor allem in Nordlagen und auf sanft geneigten Hängen vor -, wurde bewußt das ungewichtete Mittel gebildet. Damit werden auch Unterschiede zwischen den Einzugsgebieten wie die generelle Exposition ausgeglichen. Das führt zu geringeren Differenzen, als in den Abbildungen 4 bis 7 gezeigt wird, aber die Unterschiede bleiben erhalten. Mit der Variationsbreite der 


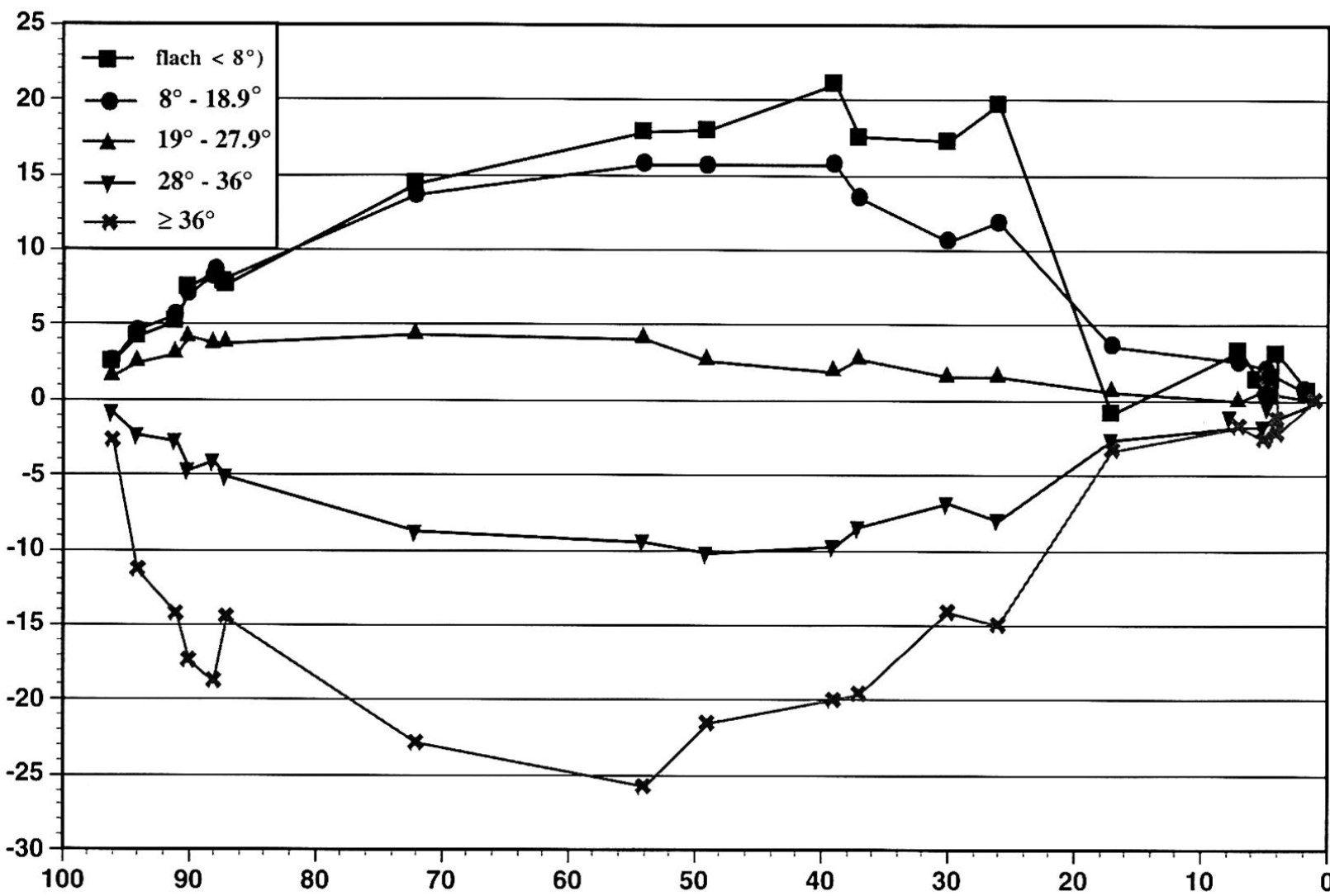

Schneebedeckung [\%] in der Höhenzonezone D (2100-2600 m ü. M.)

Abb. 7 Effekt der Hangneigung: Abweichung der Schneebedeckung nach Hangneigungsklassen vom Mittel der Höhenzone D, Einzugsgebiet Rhein-Felsberg, sechsjährige Reihe.

einzelnen Aggregationen kann nun ihre Bedeutung bezüglich der Schneedecke eingeschätzt werden. Die Schneeunterlage und die Exposition führen zu einer klaren Differenzierung bei der Ausaperung, aber die regionale Untergliederung und die Hangneigung spielen eine wichtigere Rolle. Das ist einigermaßen überraschend, wird doch der Exposition meistens eine sehr große Bedeutung zugemessen. Es scheint aber, daß der Windeinfluß in dieser Höhenlage sehr groß ist oder, anders gesagt, daß enorme Schneeverfrachtungen vorkommen, die die strahlungsbedingten Unterschiede beim Schmelzen etwas verdecken.

\section{Vergleich von Ausaperungsmustern und Permafrost}

Die Verbreitung von alpinem Permafrost kann mit einer Faustregel von HAEBERLI (1975), die in Abb. 8 a dargestellt ist, abgeschätzt werden. Sie beruht auf einer empirischen Untersuchung im Bündnerland (Gebiet zwischen Flüelapaß und Piz Grialetsch) und gilt für Hänge mit einer Neigung von $20^{\circ}$ und mehr, ohne lokale Effekte wie Lawinentätigkeit zu beinhalten. Bei der Permafrostverbreitung ist grundsätzlich eine Abhängigkeit von der potentiellen Einstrahlung zu beobachten, was auch leicht einsichtig ist: Südhänge sind in höheren Lagen eher permafrostfrei als Nordhänge. Doch daneben ist auch eine deutlich tiefer reichende Verbreitung an den Westexpositionen gegenüber den nach Osten orientierten Hängen sichtbar, für die nicht die Einstrahlung verantwortlich 


\begin{tabular}{|c|c|c|c|}
\hline & \multicolumn{3}{|c|}{$\begin{array}{c}\text { Höhenzone D } \\
\text { (2100-2600 m ü. M.) }\end{array}$} \\
\hline & $\begin{array}{l}\text { Rhein- } \\
\text { Felsberg } \\
\end{array}$ & $\begin{array}{l}\text { Rhone- } \\
\text { Sion }\end{array}$ & $\begin{array}{l}\text { Tessin- } \\
\text { Bellinzona }\end{array}$ \\
\hline Untersuchungsjahre & $\begin{array}{l}1982 \\
1985 \\
1990 \\
1992 \\
1993 \\
1994\end{array}$ & 1985 & 1994 \\
\hline $\begin{array}{l}\text { Anzahl Bilder } \\
(10 \% \leq \mathrm{S} \leq 90 \%\end{array}$ & 11 & 5 & 6 \\
\hline $\begin{array}{l}\text { Mittlere } \\
\text { Schneebedeckung } \\
\text { der SCU }\end{array}$ & $53 \%$ & $56 \%$ & $67 \%$ \\
\hline
\end{tabular}

Tabelle 1 Untersuchungsdaten im Überblick.

sein kann. Die Variabilität der Schneebedeckung wird vielmehr als Ursache dieser Differenzierung vermutet. Die Schneedecke beeinflußt die Permafrostverbreitung, weil sie ein ausgezeichneter Isolator ist und im Winter den Boden vor Auskühlung schützt. Die Bodenauskühlung wird nach HAEBERLI (1990) im Mittel um $2{ }^{\circ} \mathrm{C}$ pro Meter Schneehöhe reduziert. Eine geringmächtige Schneedecke oder das Fehlen einer Schneedecke im Hochwinter begünstigt die Bildung von Permafrost.

Da das Untersuchungsgebiet von Haeberli am Rand des Einzugsgebietes Rhein-Felsberg liegt, sollte sich dort der Zusammenhang am deutlichsten zeigen. Die vorher dargelegte Ausaperungsuntersuchung bezieht sich auf die Höhenzone 2100-2600 m, also den unteren Bereich der Permafrostverbreitung, und eignet sich daher gut zum Vergleich. Die Abb. 8b zeigt die mittlere relative Schneebedeckung in Rhein-Felsberg, differenziert nach Expositionen anhand der mehrjährigen Serie von Satellitenaufnahmen. Analog dazu stellen die Abb. $8 \mathrm{c}$ und $8 \mathrm{~d}$ die Resultate für die zwei übrigen Untersuchungsgebiete dar. Die drei Abbildungen sind nicht gleich skaliert, da die Aufnahmen in den einzelnen Regionen nicht zu den gleichen Zeitpunkten erfolgten und die Auswertungen also nur relativ miteinander verglichen werden können.

Abb. 8 b zeigt anhand der relativen Schneebedeckung, daß in Rhein-Felsberg alle Westexpositionen früher ausapern als die entsprechenden Osthänge, wie es auch in Abb. 6 ersichtlich ist. Somit kann auf eine deutlich gerin-

\begin{tabular}{|c|c|c|c|}
\hline Aggregationen & $\begin{array}{l}\text { Abwei } \\
\text { Schnee } \\
\text { in } \\
\text { Rhein- } \\
\text { Felsberg } \\
\text { S (\%) }\end{array}$ & $\begin{array}{l}\text { ichung vo } \\
\text { bedecku } \\
\text { Höhenz } \\
\text { 00-2600 } \\
\text { Rhone- } \\
\text { Sion } \\
\text { S (\%) }\end{array}$ & $\begin{array}{l}\text { mittlerer } \\
\text { g der SCU } \\
\text { ne D } \\
\text { ü. M.) } \\
\text { Tessin- } \\
\text { Bellinzona } \\
\text { S (\%) }\end{array}$ \\
\hline Gletschera & $+20^{b}$ & +9 & $+12^{b}$ \\
\hline $\begin{array}{l}\text { Permafrost } \\
\text { wahrscheinlich }^{a}\end{array}$ & 0 & -3 & -1 \\
\hline Vegetationslos $^{a}$ & -3 & -1 & -3 \\
\hline Restklasse $^{a}$ & -12 & -5 & -8 \\
\hline $\begin{array}{l}\text { Variationsbreite } \\
\text { Schneeunterlagen }\end{array}$ & 32 & 14 & 20 \\
\hline Gotthardgebiet & +12 & +16 & +15 \\
\hline $\begin{array}{l}\text { Vorderrhein / Wildh. / } \\
\text { Leventina }\end{array}$ & -1 & +15 & +2 \\
\hline $\begin{array}{l}\text { Mittelbünden / Goms / } \\
\text { Riviera }\end{array}$ & -6 & +1 & -8 \\
\hline $\begin{array}{l}\text { Oberhalbstein / } \\
\text { Rhonetal /- }\end{array}$ & -6 & -8 & - \\
\hline -/Walliser Alpen /- & - & -25 & - \\
\hline $\begin{array}{l}\text { Variationsbreite } \\
\text { Region }\end{array}$ & 18 & 41 & 33 \\
\hline Nord & +5 & +5 & +9 \\
\hline Nordost & +7 & +5 & +6 \\
\hline Ost & +8 & +4 & +4 \\
\hline Südost & +4 & +3 & -3 \\
\hline Süd & -6 & -8 & -9 \\
\hline Südwest & -10 & -10 & -10 \\
\hline West & -10 & -8 & -5 \\
\hline Nordwest & -2 & -2 & +3 \\
\hline $\begin{array}{l}\text { Variationsbreite } \\
\text { Exposition }\end{array}$ & 18 & 15 & 19 \\
\hline$<8^{\circ}$ & +13 & +9 & +2 \\
\hline $8-18,9^{\circ}$ & +10 & +7 & +5 \\
\hline $19-27,9^{\circ}$ & +5 & +3 & +4 \\
\hline $28-35,9^{\circ}$ & -4 & -4 & 0 \\
\hline$\geq 36^{\circ}$ & -14 & -16 & -11 \\
\hline $\begin{array}{l}\text { Variationsbreite } \\
\text { Hangneigung }\end{array}$ & 27 & 25 & 16 \\
\hline
\end{tabular}

a interpoliert auf 2700 m ü. M. $\quad$ b inkl. blankes Eis

Tabelle 2 Abweichung der relativen Schneebedeckung (S) vom Durchschnitt aller Schneebedeckungseinheiten (SCU) für verschiedene Schneeunterlagen, Regionen, Expositionen und Hangneigungen der Höhenzone D (2100-2600 m ü. M.). 

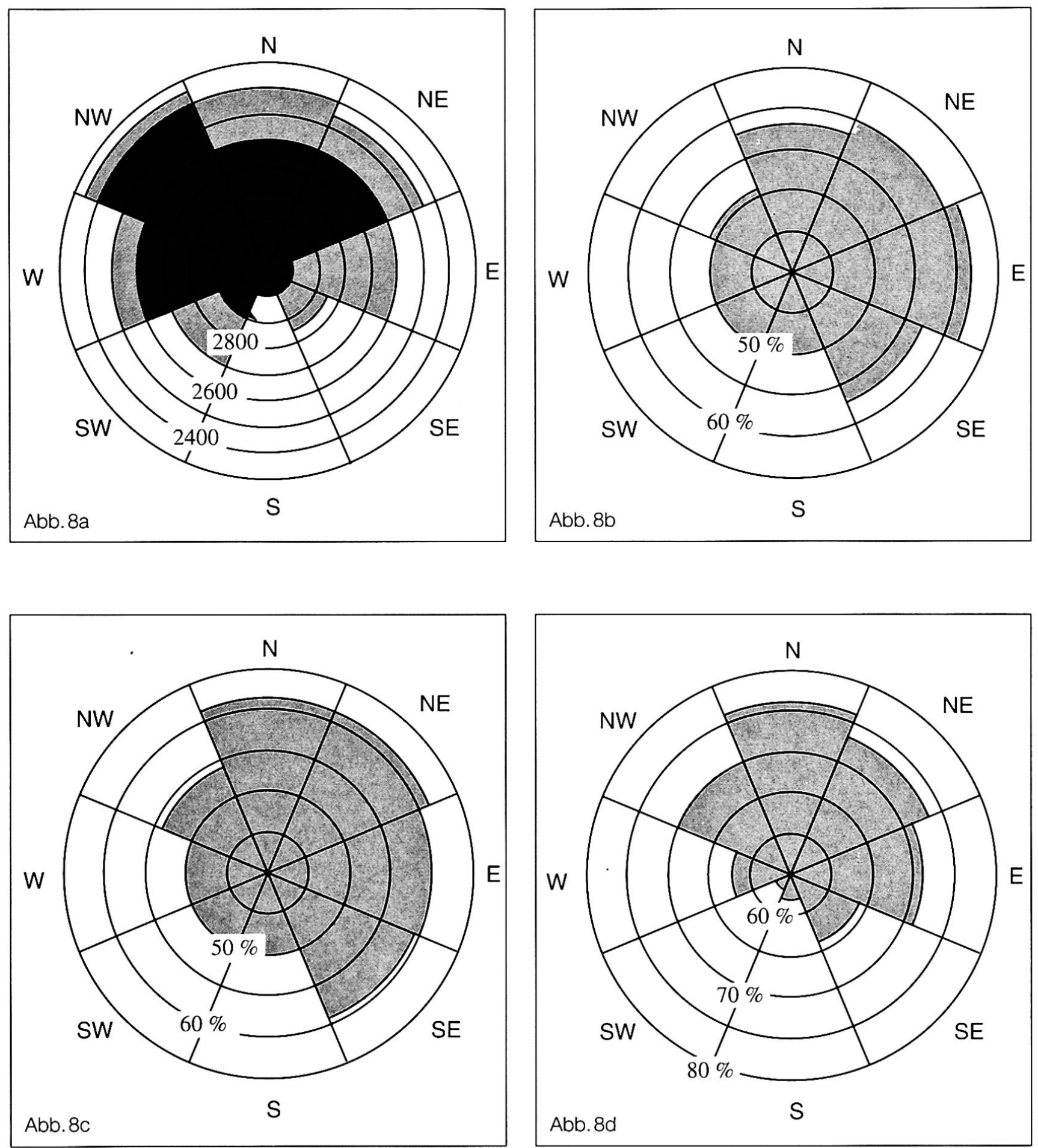

Abb. 8 a) Permafrostverbreitung (m ü. M.) nach W. HAEBERLI (1975). Dunkel schattiert ist der Bereich von wahrscheinlichem Permafrost, hell schattiert von möglichem Permafrost.

b-d): Mittlere relative Schneebedeckung nach Exposition in Höhenzone D während der Schneeschmelze: b) Einzugsgebiet Rhein-Felsberg, sechsjährige Reihe, c) Rhone-Sion, 1985, d) Ticino-Bellinzona, 1994. 
gere Schneemächtigkeit in den Westexpositionen geschlossen werden. Die geringe Schneebedeckung wird dadurch als beeinflussender Faktor für den tiefreichenden Permafrost an den Westhängen bestätigt. An den ostexponierten Hängen wurde der Triebschnee aus den Westhängen abgelagert, was zu überdurchschnittlichen Schneehöhen und, wie Abb. 8 b zeigt, zu einer späteren Ausaperung führt. Die Permafrostgrenze liegt an den Osthängen vergleichsweise hoch, was bedeutet, daß der Effekt einer guten Isolation im Winter durch eine mächtige Schneedecke dominanter ist als ein verkürzter Einstrahlungszeitraum durch späte Ausaperung im Sommer. Die Ausaperung nach Exposition ist in den anderen zwei Untersuchungsgebieten leicht verändert, wie die Abb. $8 \mathrm{c}$ und $8 \mathrm{~d}$ zeigen: In Rhone-Sion und Ticino-Bellinzona weisen die Südwesthänge eine minimale relative Schneebedeckung auf, während das Maximum im Bereich Nordost bis Nord liegt. Wie bereits erwähnt, sind die Hauptwindrichtung bzw. die damit verbundenen Schneeverfrachtungen die Ursache für diese Variabilität in der Schneebedeckung. Somit ist zu vermuten, daß sich in diesen zwei Regionen auch die Permafrostverbreitung leicht verändert darstellt, indem die tiefstgelegenen Vorkommen eher an Nordexpositionen zu erwarten sind. Insgesamt zeigt der Vergleich zwischen Permafrostvorkommen und mittlerer Schneebedeckung an westlichen und östlichen Hängen ein gegenläufiges Verhalten von Schneebedeckung und Permafrostvorkommen. An südlich orientierten Hängen sind ein Zurücktreten von Permafrost und Schneedecke sowie an nördlich orientierten Hängen ein verstärktes Auftreten von Permafrost und eine langanhaltende Schneebedeckung erkennbar. Ein wechselseitiger Zusammenhang zwischen Schneebedekkung und Permafrostverbreitung ist deutlich: Die Schneedecke schützt den Boden im Winter vor Auskühlung, andererseits findet auf dem Permafrostboden und den vegetationslosen Gebieten eine spätere Ausaperung statt, wie Tabelle 2 zeigt. Die gezeigte Variabilität der Schneebedeckung zwischen den drei Untersuchungsgebieten läßt allerdings vermuten, daß sich die Faustregel der Permafrostverteilung, die HAEBERLI für das Gebiet zwischen Flüelapaß und Piz Grialetsch (GR) erstellt hat, auf andere Alpenregionen nicht beliebig übertragen läßt.

\section{Literatur}

DOZIER, J. (1989): Spectral Signature of Alpine Snow Cover from the Landsat Thematic Mapper. Remote Sens. Environ., Vol. 28, 9-22.

EHRLER, C., SEIDEL, K. (1995): Mutual effects of the climate change and the alpine snow cover and their influence on the runoff regime evaluated with the aid of satellite remote sensing. In: Stein, T. I., editor, IGARSS '95; Quantitative Remote Sensing for Science and Applications, Florence, Italy, Vol. 3, 1973-1975.

HAEBERLI, W. (1975): Untersuchungen zur Verbreitung von Permafrost zwischen Flüelapaß und Piz Grialetsch (GR). Mitteilungen der Versuchsanstalt für Wasserbau, Hydrologie und Glaziologie ETH Zürich 17, 221 p.

HAEBERLI, W. (1990): Permafrost. In: Schnee, Eis und Wasser der Alpen in einer wärmeren Atmosphäre. Internationale Fachtagung Mai 1990 an der VAW ETH Zürich. Mitteilungen der Versuchsanstalt für Wasserbau, Hydrologie und Glaziologie ETH Zürich 108, 71-88.

HALL, D. K., MARTINEC, J.(1985): Remote Sensing of Ice and Snow. Chapman and Hall; London, New York.

KELLER, F. (1994): Interaktion zwischen Schnee und Permafrost. Mitteilungen der VAW-ETH Zürich, Nr. 127, 145 Seiten. KILLINGTVEID, Å., SAND, K. (1991): Areal Distribution of Snowcover in a Mountainous Area. Proceedings of Northern Hydrology Symposium, July 10-12, 1990, Saskatoon, Saskatchewan, Canada, NHRI Symposium, No. 6, 189-203.

RANGO, A. (1985): Assessment of Remote Sensing Input to Hydrologic Models. Water Resources Bulletin, Vol. 21, No. 3 , 423-432.

ROSENTHAL, W. (1996): Estimating Alpine Snow Cover with Unsupervised Spectral Unmixing. In: Stein, T. I., editor, IGARSS '96; Remote Sensing for a Sustainable Future, Lincoln, USA, Vol. 4, 2252-2254.

SCHWEIZER, J. (1996): Bildung und Eigenschaften der Schneedecke mit einem Seitenblick auf die Lawinenbildung. Die Alpen, 1/96, 12-18.

SEIDEL, K., EHRLER, C., MARTINEC, J. (1996): Multisensor Analysis of Satellite Images for Regional Snow Distribution. In: Progress in Environmental Research and Applications, 15th EARSeL Symposium 1995, Basel, Switzerland, 213-220.

WITMER, U. (1984): Eine Methode zur flächendeckenden Kartierung von Schneehöhen unter Berücksichtigung von reliefbedingten Einflüssen. Geographica Bernensia, G 21, Geographisches Institut Universität Bern, 140. 